\title{
Overall clinical and trichoscopic analysis performed in patients who underwent pressurized intraperitoneal aerosol chemotherapy (PIPAC) treatment for peritoneal carcinomatosis - initial trial preliminary report
}

\author{
Maciej Nowacki ${ }^{1}$, Katarzyna Nowacka², Iwona Głowacka ${ }^{1}$, Barbara Zegarska², Wojciech Zegarski ${ }^{1}$
}

${ }^{1}$ Chair and Department of Surgical Oncology, Ludwik Rydygier's Collegium Medicum in Bydgoszcz, Nicolaus Copernicus University in Torun, Oncology Centre - Prof. Franciszek Łukaszczyk Memorial Hospital in Bydgoszcz, Poland

${ }^{2}$ Chair of Cosmetology and Aesthetic Dermatology, Ludwik Rydygier's Collegium Medicum in Bydgoszcz, Nicolaus Copernicus University in Torun, Poland

Adv Dermatol Allergol 2019; XXXVI (4): 461-467

DOI: https://doi.org/10.5114/ada.2018.77096

\begin{abstract}
Introduction: Cutaneous adverse events are among the remaining problematic issues of current oncology. The term peritoneal carcinomatosis (PC) refers to the advanced cancer stage. The innovative treatment of PC includes the use of pressurized intraperitoneal aerosol chemotherapy (PIPAC).

Aim: To present a preliminary report from an initial trial aimed at an overall clinical and trichoscopic analysis performed in patients who underwent PIPAC treatment due to PC.

Material and methods: For all steps of this study we obtained the consent of the local bioethics commission \#KB 196/2018. Three different hair assessment methods were used in our study: 1) general clinical and patient self-feeling assessment; 2) hair pull test; 3 ) and trichoscopic analysis.

Results: No hair or scalp disorders were noted in the observation period. In the self-feeling test assessment the vast majority recognized their hair as being of comparable quality or even better in quality compared to previous forms of chemotherapy they had undergone. In all patients we observed a reduction of hair loss in the pull test in the hospitalization period. In trichoscopic analysis we found all determinants and signs of hair disorders in the assessed group.

Conclusions: The PIPAC is safe and is not a burdensome or aggressive form of therapy, especially according to the very important factors influencing the potential quality of hair and hair loss. The authors, however, realize that to obtain comprehensive results and evaluate this novel and promising method we need to perform more research without any limitations like those in our study.
\end{abstract}

Key words: hair loss, chemotherapy, pressurized intraperitoneal aerosol chemotherapy, drug delivery, peritoneal carcinomatosis.

\section{Introduction}

The incidence of different types of cutaneous adverse events occurring after implementation of several types and schemes of chemotherapy is one of the remaining problematic issues of current oncology, surgical oncology and dermatology [1, 2]. This multidisciplinary unresolved clinical problem is also associated with the very frequently diagnosed chemotherapy-induced hair loss (CIA) [3]. Anagen effluvium (AF) is considered synony- mous with $\mathrm{CIA}$ [4]. Such type of effluvium means active hair loss of more than 100 hairs/day over a time period of 2-4 weeks. Many authors describe AF as a consequence of direct toxicity to the highly proliferative matrix keratinocytes, as well as the follicular pigmentary system. It is hypothesized that hair matrix and dermal papilla are very sensitive to toxins, and some chemotherapeutics may lead to rapid apoptosis [5]. This clinical issue is very important because it is estimated that around $8 \%$ of pa-

Address for correspondence: Maciej Nowacki, Chair and Department of Surgical Oncology, Ludwik Rydygier's Collegium Medicum, Nicolaus Copernicus University, Oncology Centre - Prof. Franciszek Łukaszczyk Memorial Hospital, 2 Romanowskiej St, 85-796 Bydgoszcz, Poland, phone: +48 5237434 20, fax: +48 5237434 12, e-mail: maciej.s.nowacki@gmail.com Received: 30.04.2018, accepted: 14.06.2018. 
tients reject chemotherapy to avoid this distressing factor strongly influencing quality of life [6]. Not only hair loss and chemotherapy-induced alopecia (CIA), which has been studied since the early 1960s, is the main adverse toxic effect of a large group of currently used oncostatic agents. The other adverse events include direct macroscopic and microscopic changes, from the hair condition, color or texture to specific changes of hair follicles and hair structure [7-9]. Currently many such changes could be analyzed in assessment of qualitative and quantitative parameters very often supported by evaluation of trichological parameters [10]. To date there are many published data presenting potential pathobiological factors playing a role in $\mathrm{CIA}$ and other chemotherapy-induced hair disorders as well as papers focusing on possible preventive or therapeutic strategies, but both topics still remain unexplained $[6,11,12]$. The other problem is the fact that most published data refer only to the different aspects of hair quality in patients who underwent classic intravenous (i.v.) forms of chemotherapy, but there is still a lack of publications related to the other medical forms of chemotherapeutics delivery [13, 14].

A very specific group of oncological patients includes those suffering from peritoneal carcinomatosis (PC), in which mainly the different types of intraperitoneal forms of chemotherapy implementation to achieve a higher drug concentration in the peritoneal cavity are used [15] One such treatment technique in recent years, proposed by the scientific team of Prof. Reymond, is the innovative pressurized intraperitoneal aerosol chemotherapy (PIPAC) [16]. The method involves the combination of laparoscopic miniinvasive surgical techniques with a modern way of delivering the drug in the form of appropriately dispersed drops of aerosol under pressure [17].

\section{Aim}

The aim of our study was to present the scientific preliminary report from the initial trial aimed in our center at an overall clinical and trichoscopic analysis performed in patients who underwent PIPAC treatment due to diagnosed peritoneal carcinomatosis. The patients qualified for PIPAC therapy very often have advanced stage IV cancer in which all of the aspects of quality of life related to the used therapeutic method play a key role in their treatment $[18,19]$. Therefore in our study we focus on a very important aspect related to the detailed assessment of hair condition and its potential loss after PIPAC therapy.

\section{Material and methods \\ Patients}

Our study included patients with clinically confirmed PC. All patients were in a good clinical condition without any contraindications for PIPAC treatment. All patients were always consulted by a clinical oncologist before procedure implementation. All patients had undergone concomitant neo-adjuvant systemic therapy and experienced hair loss in the past. All patients gave voluntary and informed consent to the planned treatment, and the study was performed in accordance with the precepts established by the Declaration of Helsinki. For all steps of this study we obtained the consent of the local bioethics commission \# KB 196/2018.

\section{PIPAC procedure}

The PIPAC procedure was performed in all patient under general anesthesia. Always two double secured blunt trocars (Kii Balloon Blunt TIP - Applied Medical, CA, USA) were used after previously performed standard 12 mm Hg capnoperitoneum using a Veress needle. In all cases diagnostic laparoscopy combined with local peritonectomy and sample collection for pathology assay was performed after previous PCI index measurement. After full diagnostic assurance that the PIPAC procedure could be safely performed and after checking the completeness of the equipment, the micro-pump/nebulizer (Capnopen, Capnomed, Villingendorf, Germany) was precisely inserted into one trocar, and a standard $10 \mathrm{~mm} \mathrm{30^{ \circ }}$ laparoscopic camera (Olympus, Japan) was inserted into the opposite second trocar to achieve control visualization. In all patients the standard chemotherapy regimen recommended by other PIPAC centers was used. A pressurized aerosol containing chemotherapy agents - cisplatin $\left(7.5 \mathrm{mg} / \mathrm{m}^{2}\right.$ in $\left.150 \mathrm{ml} 0.9 \% \mathrm{NaCl}\right)$ immediately followed by doxorubicin $\left(1.5 \mathrm{mg} / \mathrm{m}^{2}\right.$ in $\left.50 \mathrm{ml} 0.9 \% \mathrm{NaCl}\right)$ agents - was implemented via a nebulizer. We always used the 6- to 8-week mean time period between standard chemotherapy and PIPAC [20, 21].

\section{Clinical and trichoscopic analysis}

In our study we performed three different types of trichological analysis. The first one was the general clinical hair macroscopic inspection including the assessment of hair quality, dye, scalp quantity and distribution as well as patient self-feeling and subjective hair assessment. The second assay was based on the hair pull test. This part of the assessment always included pulling 50-60 hairs using slow traction from the frontal, parietal and temporal area.

The study material in these two sections was analyzed at admission to the hospital and after PIPAC treatment implementation when the patient was discharged from the hospital. The study limitation in our protocol was the fact that in our initial study we performed the trichoscopic assessments only after 2 weeks, not in 4, because of the Polish PIPAC program organization.

The last, third diagnostic method was associated with performance of the trichoscopy using the LED dermatoscope (HEINE DELTA 20 T - Heine Germany) - (40x 
magnification). This part of the assessment was always performed on the day of discharge from hospital. The research methodology was based on modern literature data, recommendations and current guidelines [22].

\section{Results}

A total of 6 patients $(n=6 / 100 \%)$ were analyzed in our study. The gender distribution was equal -3 men and 3 women (50:50) with an average age equal to 52 years (range: 41-68 years). The median value of the measured Karnofsky index (KI) in this group was around 86 points (range: 59-98 points). In all patients the peritoneal carcinomatosis index $(\mathrm{PCl})$ was measured during the procedure. The mean value of $\mathrm{PCI}$ was 19 points and the range was $17-26$ points. All of the patients analyzed in the study suffered from PC of gastric cancer origin. None of the analyzed patients had undergone a previous gastrectomy or any radical type of curatively targeted therapy and had been classified as non-resectable. All of the patients have in their medical history undergone concomitant neo-adjuvant systemic therapy. In all treated patients ascites has been diagnosed. The mean amount was around $867 \mathrm{ml}$ (range: 51-3198 ml). Detailed patient characteristics are presented in Table 1.

In all patients the PIPAC procedure was performed successfully. During all procedures all of the steps were performed according to the PIPAC standards and guidelines. No intraoperative or postoperative complications or adverse events were observed. The median hospital stay after the procedure was 3 days (min./max.: 3-5). In all cases the general hospitalization time was around seven days. All the patients were discharged from hospital in a good general condition.

\section{Clinical and trichoscopic analysis}

In the first part of the analysis the general clinical hair macroscopic inspection was always performed by the same physician. No pathological changes were noted in the standard general clinical assessment between admission and home discharge time in hair quality or hair color, and no alarming symptoms were observed according to the scalp quality and its disorders or in general (first-line) macroscopic hair distribution analysis. In this part of the study patient self-feeling and subjective hair assessment were considered very important aspects of the analysis. The patients completed the seven-point visual scale in which they assessed subjectively the quality of their hair on admission and at discharge. Two patients classified their hair as in very bad condition (33.4\%), two as in bad condition (33.4\%), one as hair in which no changes were noticed to the condition before the cancer diagnosis (16.7\%), and one person classified their hair as in good condition (16.7\%). In comparison, in the same test performed after the PIPAC procedure implementation on the day of patient discharge, in 2 patients no changes were noticed (33.4\%), 3 (50\%) patients stated that they were in good condition compared to previous forms of drug delivery, and 1 person reported that her hair was in very good condition (16.7\%) (Table 2). From all the patients in the group of women (50\%), better subjective opinions about their hair were expressed than in the group of men.

In the second assay based on the hair pull test the extracted hairs were counted excluding broken hairs. In the assessment performed just after admission in 2 (33.4\%) patients positive results were obtained (10 and 17 points). The same number of patients obtained positive results (11 and 10 points). In all patients there were more than 2 hairs found before and after treatment in each assessment. In all patients we observed reduction of hair loss in the pull test in the hospitalization period. Detailed results are presented in Table 3.

In the performed trichoscopic analysis telogen alopecia was observed in $1(16.7 \%)$ case. In this case all clinical and dermatosopic findings related to alopecia including hair density with presence of empty follicles

Table 1. Patients' $(n=6)$ characteristics treated by pressurized intraperitoneal aerosol chemotherapy (PIPAC) for advanced peritoneal carcinomatosis

\begin{tabular}{lc}
\hline Parameter & Value \\
\hline Gender: & $3(50 \%)$ \\
\hline Female & $3(50 \%)$ \\
\hline Male & 52 \\
\hline Age: & $41-68$ \\
\hline Median & 86 \\
\hline Range & $59-98$ \\
\hline Karnofsky index: & 19 \\
\hline Mean & $17-26$ \\
\hline Range & \\
\hline Peritoneal carcinomatosis index (PCI) measured during PIPAC \\
procedure: \\
\hline Mean & 667 \\
\hline Range & $6(100 \%)$ \\
\hline Cancer origin: & $0(0 \%)$ \\
\hline Gastric cancer & \\
\hline Previous gastrectomy/radical (curative) \\
\hline surgery
\end{tabular}


Table 2. Patient self-feeling and subjective hair assessment test

A

\begin{tabular}{ccccccc} 
The worst & Very bad & Bad & Nothing & Good & Very good & Best \\
\hline $0 \%$ & $33.4 \%$ & $33.4 \%$ & $16.7 \%$ & $16.7 \%$ & $0 \%$ & $0 \%$ \\
\hline
\end{tabular}

B

\begin{tabular}{ccccccc} 
The worst & Very bad & Bad & Nothing & Good & Very good & Best \\
\hline $0 \%$ & $0 \%$ & $0 \%$ & $33.4 \%$ & $50 \%$ & $16.7 \%$ & $0 \%$ \\
\hline
\end{tabular}

Patient self-feeling and subjective hair assessment test, performed just after admission (A) and after PIPAC procedure implementation on the day of patient discharge $(B)$.

Table 3. Hair pull-test analysis

\begin{tabular}{lcc}
\hline $\begin{array}{l}\text { Number of } \\
\text { patient }\end{array}$ & $\begin{array}{c}\text { Measurement } \\
\text { performed just after } \\
\text { admission (all areas) }\end{array}$ & $\begin{array}{c}\text { Measurement } \\
\text { performed after PIPAC } \\
\text { (all areas) }\end{array}$ \\
\hline 1 & 6 & 4 \\
\hline 2 & 9 & 8 \\
\hline 3 & 10 (positive) & 11 (positive) \\
\hline 5 & 4 & 1 \\
\hline 6 & 9 & 6 \\
\hline
\end{tabular}

The second trichologic assay was based on the hair pull test. This part of assessment has always included pulling of 50-60 hairs using slow traction from the frontal, parietal and temporal area - where the extracted hairs were counted excluding broken hairs: - just after admission and after the PIPAC procedure on the day of discharge from hospital.

Table 4. Trichoscopic analysis

\begin{tabular}{lc}
\hline Alopecia & $16.7 \%$ \\
\hline Thinning hairs & $66.8-83.5 \%$ \\
\hline Tapering hairs & $83.5 \%$ \\
\hline Regrowing hairs & $16.7 \%$ \\
\hline Pohl-Pinkus hairs & $40-83.5 \%$ \\
\hline Exclamation mark hairs & $16.7 \%$ \\
\hline
\end{tabular}

In trichoscopic analysis alopecia was observed in $16.7 \%$ of patients. Thinning hair was observed 66.8-83.5\%, tapered hair in $83.5 \%$, regrowing hairs in $16.7 \%$, Pohl-Pinkus constrictions in 40-83.5\%, and exclamation mark hairs in $16.7 \%$ of patients.

as well as absence of hair shaft diameter variation and peripilar halo were found. Thinning hair was observed in $66.8-83.5 \%$ of patients (Figure $1 \mathrm{~A}$ ), tapered hair in $83.5 \%$ (Figure $1 \mathrm{~B}$ ), regrowing hairs in $16.7 \%$, Pohl-Pinkus constrictions in 40-83.5\%, and exclamation mark hairs in $16.7 \%$ patients respectively. All the obtained results are presented in Table 4.
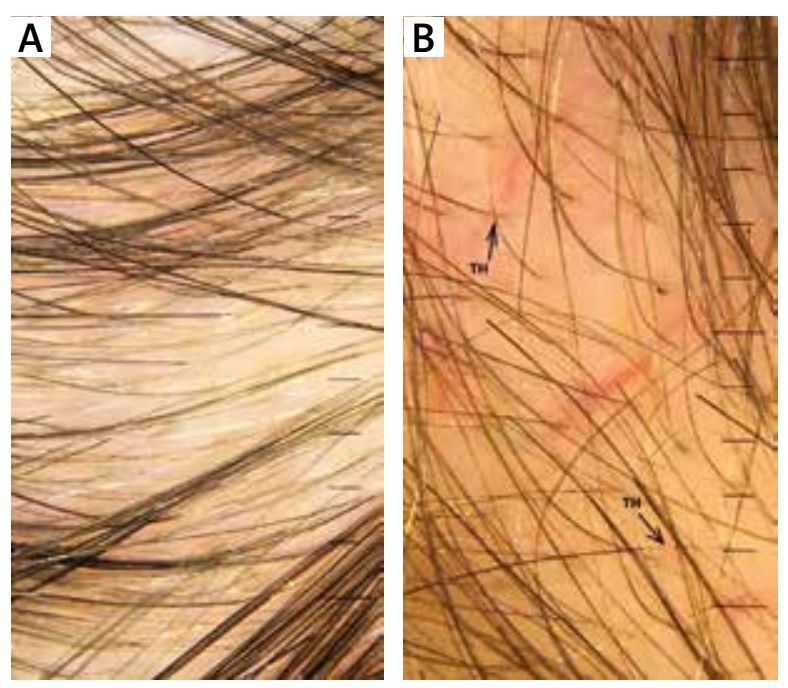

Figure 1. Visualization of hairs in trichoscopy: $\mathbf{A}$ - thinning hairs; $\mathbf{B}$ - tapered hairs. LED dermatoscope (HEINE DELTA $20 \mathrm{~T}$ - Heine Germany)

\section{Discussion}

The term peritoneal carcinomatosis refers to the metastatic phenomenon of uncontrolled, rapid tumor growth within the peritoneal cavity most often of ovarian or gastro-intestinal (as well as a representative group of 'other') origin including primary types of cancer [23, 24]. Usually PC has a very poor prognosis and it is invariably terminal [25]. It may be diagnosed during postoncological treatment as a form of rapid cancer progression or in many cases it can be diagnosed at the same time as detection of the primary cancer $[26,27]$. The PC is mainly manifested by several clinically important signs and symptoms such as ascites, diarrhea, abdominal pain, bloating, weight loss, high risk of bowel obstruction and possible perforation. The PC could also lead in time to profound cachexia and inanition [28, 29]. For many years PC has been one of most problematic issues of surgical oncology and oncology. That is why, especially for the 
last three decades since Lambert and latterly Sugerbaker described the combination technique of cytoreduction (CRS) and hyperthermic intraperitoneal chemoperfusion (HIPEC), the topic of effective peritoneal carcinomatosis treatment is still increasingly developing globally [30]. Despite the fact that CRS + HIPEC in many types of PC is currently a gold standard, there is still a group in which this method could not be used mainly because it is too aggressive, because of the poor clinical patient condition or too extensive disease progression (measurable using e.g. peritoneal cancer index-PCI) [31-33]. For these patients for many years only palliative systemic chemotherapy was available, which was considered to have many adverse effects including pathological skin manifestations or hair loss and their different structure and quality disorders $[34,35]$. That is why currently more often in this group of patients PIPAC surgical drug delivery, first implemented in November 2011 in Herne, Germany by Nowacki et al., is used and recommended [36]. PIPAC drug delivery, based on a mini-invasive type of surgery (laparoscopy), has several described potential benefits for patients including better inner organs, peritoneal cavity and tumor tissue penetration and lower chemotherapeutic doses than those used in other forms of delivery, resulting in significantly lower toxicity. The PIPAC therapy could be finally implemented safely as multiple form of therapy in 1 patient [37].

To date, to our best knowledge there are no published data specifically aiming at aspects of hair loss and hair disorders in patients who underwent PIPAC surgery. As mentioned before, the quality of life is in the group of patients with diagnosed PC one of the key points and main aspects of any potentially planned course of treatment [38]. Therefore in our study we focus on a very important aspect related to the detailed hair condition assessment and its potential loss after PIPAC therapy. That is why we have focused on overall trichoscopic analysis supported by simple but effective clinical tests including clinical inspection and the pull test. All of these tools are currently recommended and by many authors are considered as indispensable tools in differential diagnosis of hair and scalp diseases [39, 40].

At the beginning of our study we found one problematic point of our evaluation, i.e. the fact that almost all patients with PC are patients who have a previous oncological treatment history including mainly, as in our case, different i.v. forms of therapy [41]. The potential result masking effect was limited in our study due to the use of "sandwich" forms of therapy in our center proposed in the literature by Alyami et al., which is based on multimodal treatment scheduled in the time intervals allowing one to assess only the condition after the PIPAC treatment [42]. However, this problem was limited due to the fact that patients were diagnosed at admission and at discharge to home. In our initial study we assessed 6 persons suffering from PC of gastric cancer origin in an advanced stage (PCI 17-26). None of these patients had previously undergone any type of radical (curative) form of surgical treatment.

Very interesting preliminary results were obtained in the patient self-feeling and subjective hair assessment test in which patients subjectively recognized a better general hair condition than after immediate post-treatment stages not related to the intraperitoneal drug delivery. This situation could have resulted from the better drug concentration and local dose enhancement in the peritoneal cavity with simultaneous lower general toxicity after PIPAC treatment proved by many other authors [43-45].

In the hair-pull test we found that in all patients the number of extracted hairs was slightly lower after PIPAC therapy than on the day of patient admission to the hospital. In our opinion it is not an argument for the statement that PIPAC therapy improves the hair quality and limits its loss, but we consider this situation with normal hair loss stabilization observed in time periods where i.v. chemotherapy is not implemented $[46,47]$. We can however conclude that PIPAC therapy is safe and does not negatively affect the normal process of hair regrowth after previous systemic chemotherapy.

Although trichoscopic features indicating an active dystrophic mechanism of hair loss were seen in all patients, this is probably the effect of previous standard intravenous chemotherapy. Based on clinical assessment and improvement in the pull test, it can be concluded that PIPAC therapy does not aggravate dystrophic hair loss caused by previous intravenous chemotherapy. We confirmed in our study all kinds of signs and diagnostic images found in standard trichoscopy criteria including alopecia, thinning hairs, tapering hairs, regrowing hairs, Pohl-Pinkus hairs, and exclamation mark hairs [48, 49]. This situation could be related to the fact that PIPAC therapy is a safe form of local chemotherapy delivery but still it is a form of treatment using highly cytotoxic compounds.

Our study is one of the first in which the topic of PIPAC treatment and important aspects of hair loss and hair disorders are discussed. The authors are aware of all of this trial's limitations, in particular related to the small number of patients, relatively short time of observation the masking effect of earlier stages of systemic treatment as well as limitations in available trichoscopy produceres. However, we are sure that these initial results will fully contribute to further more advanced work on this subject.

Pressurized intraperitoneal aerosol chemotherapy treatment is a very promising form of therapy in patients suffering from peritoneal carcinomatosis who do not have a possibility for any other form of therapy. In our initial trial we also found that PIPAC is safe and is not a form of burden or aggressive therapy especially according to the very important factors influencing quality of life after oncological intervention, including the potential 
quality of hair and hair loss. The authors, however, realize that to obtain comprehensive results and evaluate this novel and promising method we need to perform more research in particular on wider groups and the analysis of many other factors without any limitations like those in our study.

\section{Conflict of interest}

The authors declare no conflict of interest.

\section{References}

1. Owczarek W, Słowińska M, Lesiak A, et al. The incidence and management of cutaneous adverse events of the epidermal growth factor receptor inhibitors. Adv Dermatol Allergol 2017; 34: 418-28.

2. Sammut SJ, Tomson N, Corrie P. Pyogenic granuloma as a cutaneous adverse effect of vemurafenib. N Engl J Med 2014; 371: 1265-7.

3. Komen MMC, van den Hurk CJG, et al. Patient-reported outcome assessment and objective evaluation of chemotherapy-induced alopecia. Eur J Oncol Nurs 2018; 33: 49-55.

4. Kanwar AJ, Narang T. Anagen effluvium. Indian J Dermatol Venereol Leprol 2013; 79: 604-12.

5. Rubio-Gonzalez B, Juhász M, Fortman J, Mesinkovska NA. Pathogenesis and treatment options for chemotherapyinduced alopecia: a systematic review. Int J Dermatol 2018 In press doi: 10.1111/jdv.14970.

6. Paus R, Haslam IS, Sharov AA, Botchkarev VA. Pathobiology of chemotherapy-induced hair loss. Lancet Oncol 2013; 14: e50-9.

7. Muth CC. Chemotherapy and Hair Loss. JAMA 2017; 317: 656

8. Bodó E, Tobin D, Kamenisch Y, et al. Dissecting the impact of chemotherapy on the human hair follicle: a pragmatic in vitro assay for studying the pathogenesis and potential management of hair follicle dystrophy. Am J Pathol 2007; 171: 1153-67.

9. Routhouska S, Gilliam AC, Mirmirani P. Hair depigmentation during chemotherapy with a class III/V receptor tyrosine $\mathrm{ki}$ nase inhibitor. Arch Dermatol 2006; 142: 1477-9.

10. Kanti V, Nuwayhid R, Lindner J, et al. Analysis of quantitative changes in hair growth during treatment with chemotherapy or tamoxifen in patients with breast cancer: a cohort study. Br J Dermatol 2014; 170: 643-50.

11. Brower V. Scalp cooling and hair loss during breast cancer chemotherapy. Lancet Oncol 2017; 18: e199.

12. Rice BA, Ver Hoeve ES, DeLuca AN, et al. Registry study to assess hair loss prevention with the Penguin Cold Cap in breast cancer patients receiving chemotherapy. Breast Cancer Res Treat 2018; 167: 117-22.

13. Ishitobi M, Shibuya K, Komoike Y, et al. Preferences for oral versus intravenous adjuvant chemotherapy among early breast cancer patients. Patient Prefer Adherence 2013; 7: 1201-6.

14. Raavé R, de Vries RB, Massuger LF, et al. Drug delivery systems for ovarian cancer treatment: a systematic review and meta-analysis of animal studies. Peer J 2015; 3: 1489.

15. Murono K, Kawai K, Hata K, et al. Regimens of intraperitoneal chemotherapy for peritoneal carcinomatosis from colorectal cancer. Anticancer Res 2018; 38: 15-22.

16. Nowacki M, Peterson M, Kloskowski T, et al. Nanoparticle as a novel tool in hyperthermic intraperitoneal and pres- surized intraperitoneal aerosol chemotheprapy to treat patients with peritoneal carcinomatosis. Oncotarget 2017; 8: 78208-24.

17. Göhler D, Große S, Bellendorf A, et al. Hyperthermic intracavitary nanoaerosol therapy (HINAT) as an improved approach for pressurised intraperitoneal aerosol chemotherapy (PIPAC): technical description, experimental validation and first proof of concept. Beilstein J Nanotechnol 2017; 8: 2729-40.

18. Loggie BW, Thomas P. Gastrointestinal cancers with peritoneal carcinomatosis: surgery and hyperthermic intraperitoneal chemotherapy. Oncology (Williston Park) 2015; 29: 515-21.

19. Geng X, Liu H, Lin T, et al. Survival benefit of gastrectomy for gastric cancer with peritoneal carcinomatosis: a propensity score-matched analysis. Cancer Med 2016; 5: 2781-91.

20. Tempfer CB, Winnekendonk G, Solass W, et al. Pressurized intraperitoneal aerosol chemotherapy in women with recurrent ovarian cancer: a phase 2 study. Gynecol Oncol 2015; 137: 223-8.

21. Cazauran JB, Alyami M, Lasseur A, et al. Pressurized intraperitoneal aerosol chemotherapy (PIPAC) procedure for nonresectable peritoneal carcinomatosis (with video). J Gastrointest Surg 2018; 22: 374-5.

22. Galliker NA, Trüeb RM. Value of trichoscopy versus trichogram for diagnosis of female androgenetic alopecia. Int J Trichol 2012; 4: 19-22.

23. Coccolini F, Gheza F, Lotti M, et al. Peritoneal carcinomatosis. World J Gastroenterol 2013; 19: 6979-94.

24. Robella M, Vaira M, Marsanic P, et al. Treatment of peritoneal carcinomatosis from ovarian cancer by surgical cytoreduction and hyperthermic intraperitoneal chemotherapy (HIPEC). Minerva Chir 2014; 69: 27-35.

25. Nowacki M, Wisniewski M, Werengowska-Ciecwierz K, et al. Nanovehicles as a novel target strategy for hyperthermic intraperitoneal chemotherapy: a multidisciplinary study of peritoneal carcinomatosis. Oncotarget 2015; 6: 22776-98.

26. van Baal JOAM, van Noorden CJF, Nieuwland R, et al. Development of peritoneal carcinomatosis in epithelial ovarian cancer: a review. J Histochem Cytochem 2018; 66: 67-83.

27. McMullen JRW, Selleck M, Wall NR, Senthil M. Peritoneal carcinomatosis: limits of diagnosis and the case for liquid biopsy. Oncotarget 2017; 8: 43481-90.

28. Tan HL, Chia CS, Tan GH, et al. Gastric peritoneal carcinomatosis - a retrospective review. World J Gastrointest Oncol 2017; 9: 121-8.

29. Zhu Y, Hanna N, Boutros C, Alexander HR Jr. Assessment of clinical benefit and quality of life in patients undergoing cytoreduction and hyperthermic intraperitoneal chemotherapy (HIPEC) for management of peritoneal metastases. J Gastrointest Oncol 2013; 4: 62-71.

30. Lambert LA. Looking up: recent advances in understanding and treating peritoneal carcinomatosis. CA Cancer J Clin 2015; 65: 284-98.

31. Vaira M, Cioppa T, DE Marco G, et al. Management of pseudomyxoma peritonei by cytoreduction + HIPEC (hyperthermic intraperitoneal chemotherapy): results analysis of a twelve-year experience. In Vivo 2009; 23: 639-44.

32. Simkens GA, Rovers KP, Nienhuijs SW, de Hingh IH. Patient selection for cytoreductive surgery and HIPEC for the treatment of peritoneal metastases from colorectal cancer. Cancer Manag Res 2017; 9: 259-66.

33. Girshally R, Demtröder C, Albayrak N, et al. Pressurized intraperitoneal aerosol chemotherapy (PIPAC) as a neoadju- 
vant therapy before cytoreductive surgery and hyperthermic intraperitoneal chemotherapy. World J Surg Oncol 2016; 14: 253.

34. Odendahl K, Solass W, Demtröder C, et al. Quality of life of patients with end-stage peritoneal metastasis treated with pressurized intraperitoneal aerosol chemotherapy (PIPAC). Eur J Surg Oncol 2015; 41: 1379-85.

35. Giger-Pabst U, Solass W, Buerkle B, et al. Low-dose pressurized intraperitoneal aerosol chemotherapy (PIPAC) as an alternative therapy for ovarian cancer in an octogenarian patient. Anticancer Res 2015; 35: 2309-14.

36. Nowacki M, Alyami M, Villeneuve L, et al. Multicenter comprehensive methodological and technical analysis of 832 pressurized intraperitoneal aerosol chemotherapy (PIPAC) interventions performed in 349 patients for peritoneal carcinomatosis treatment: an international survey study. Eur J Surg Oncol 2018; S0748-7983: 30674-7.

37. Sleeman JP. PIPAC puts pressure on peritoneal metastases from pancreatic cancer. Clin Exp Metastasis 2017; 34: 291-3.

38. Lambert LA, Wiseman J. Palliative management of peritoneal metastases. Ann Surg Oncol 2018; 25: 2165-71.

39. Rakowska A, Olszewska M, Rudnicka L. Trichoscopy of scalp dysesthesia. Adv Dermatol Allergol 2017; 34: 245-7.

40. Rudnicka L, Olszewska M, Rakowska A, Slowinska M. Trichoscopy update 2011. J Dermatol Case Rep 2011; 5: 82-8.

41. Hübner M, Grass F, Teixeira-Farinha H, et al. Pressurized intraperitoneal aerosol chemotherapy - pactical aspects. Eur J Surg Oncol 2017; 43: 1102-9.

42. Alyami M, Gagniere J, Sgarbura O, et al. Multicentric initial experience with the use of the pressurized intraperitoneal aerosol chemotherapy (PIPAC) in the management of unresectable peritoneal carcinomatosis. Eur J Surg Oncol 2017; 43: 2178-83.

43. Nadiradze G, Giger-Pabst U, Zieren J, et al. Pressurized intraperitoneal aerosol chemotherapy (PIPAC) with low-dose cisplatin and doxorubicin in gastric peritoneal metastasis. J Gastrointest Surg 2016; 20: 367-73.

44. Eveno C, Haidara A, Ali I, et al. Experimental pharmacokinetics evaluation of chemotherapy delivery by PIPAC for colon cancer: first evidence for efficacy. Pleura Peritoneum 2017; 2: 103-9.

45. Demtröder C, Solass W, Zieren J, et al. Pressurized intraperitoneal aerosol chemotherapy with oxaliplatin in colorectal peritoneal metastasis. Colorectal Dis 2016; 18: 364-71.

46. Young A, Arif A. The use of scalp cooling for chemotherapyinduced hair loss. Br J Nurs 2016; 25: 24-7.

47. Nangia J. Quality of life matters: it is time to integrate scalp cooling in routine clinical practice. J Oncol Pract 2018; 14: 157-8.

48. Williamson PJ, de Berker D. Pohl-Pinkus constrictions of hair following chemotherapy for Hodgkin's disease. Br J Haematol 2005; 128: 582.

49. Mubki T, Rudnicka L, Olszewska M, Shapiro J. Evaluation and diagnosis of the hair loss patient: part I. History and clinical examination. J Am Acad Dermatol 2014; 71: 415.e1-15. 